\title{
A novel quantitative trait locus, $q C L 1$, involved in semi-dwarfism derived from Japanese rice cultivar Nipponbare
}

\author{
Kiyosumi Hori' ${ }^{1)}$, Toshio Yamamoto*1), Kaworu Ebana ${ }^{1)}$, Yoshinobu Takeuchi ${ }^{2)}$ and Masahiro Yano ${ }^{1)}$ \\ 1) National Institute of Agrobiological Sciences, 2-1-2 Kannondai, Tsukuba, Ibaraki 305-8602, Japan \\ 2) National Institute of Crop Science, 2-1-18 Kannondai, Tsukuba, Ibaraki 305-8518, Japan
}

\begin{abstract}
To identify quantitative trait loci (QTLs) associated with culm length in Japanese japonica rice varieties, we conducted QTL analyses using a set of reciprocal backcrossed inbred lines (BILs) from crosses between Nipponbare and Koshihikari. We detected five QTLs in the two BILs that are involved in the culm-length difference between Nipponbare and Koshihikari. A QTL located on the short arm of chromosome 1, qCL1, was commonly detected near the simple sequence repeat (SSR) marker RM8068 in both BILs in three growing seasons. The Nipponbare allele of $q C L 1$ shortened from 1.9 to $3.0 \mathrm{~cm}$ of culm length. Substitution lines (SLs) of Koshihikari in which the QTL was replaced with the Nipponbare allele exhibited decreased lengths of lower internodes. $q C L 1$ was mapped within about $2.6 \mathrm{Mbp}$ in the region distal from SSR marker RM10132, suggesting $q C L 1$ differed from the dwarf or semi-dwarf genes that have been previously reported. Japanese japonica rice landraces with Nipponbare alleles of DNA markers around $q C L 1$ had significantly shorter culm length than other landraces. The results suggest that the novel allele for short culm length was originally distributed in Japanese rice landraces and has been used for introducing the semi-dwarf phenotype and improving plant architecture during practical breeding.
\end{abstract}

Key Words: Oryza sativa L., quantitative trait locus, semi-dwarf phenotype, Japanese rice landraces.

\section{Introduction}

Dwarfism is an important agronomic trait in rice because it increases lodging resistance and as a result, increases yield (Hedden 2003). More than 60 dwarf and semi-dwarf genes have previously been reported in rice (Kinoshita 1998). These genes are categorized according to the distribution patterns of the elongated internodes (Takahashi and Takeda 1969, Takeda and Takahashi 1969, Kinoshita and Shinbashi 1982). Several dwarf and semi-dwarf mutants are associated with biosynthesis of or sensitivity to two phytohormone groups, namely gibberellins and brassinosteroids (Sakamoto et al. 2006, Takeda and Matsuoka 2008). Since most of the dwarf genes exert unfavorable effects on other agronomic traits such as grain length, panicle length, and number of grains per panicle (Sakamoto et al. 2004), the only useful source of dwarf genes in practical breeding thus far is considered to be the one recessive gene semi-dwarf 1 (sd1; Hedden 2003). The $s d l$ gene was isolated by means of positional cloning and encodes a gibberellic acid 20 oxidase enzyme, which controls a step in the biosynthetic pathway of gibberellins (Sasaki et al. 2002, Monna et al. 2002, Spielmeyer et al. 2002).

The development of semi-dwarf varieties using the $s d l$ gene has significantly increased world rice production during

Communicated by K. Okuno

Received May 25, 2009. Accepted July 27, 2009.

*Corresponding author (e-mail: toshyama@affrc.go.jp) the "Green Revolution". However, the extensive use of this limited dwarfing resource may cause a bottleneck effect in the genetic background of modern rice varieties. Identifying useful new semi-dwarf or dwarf genes is thus an important goal for the development of varieties with short culms while maintaining genetic diversity during practical rice breeding.

Crop landraces are valuable genetic resources because they are considered to contain a large amount of genetic diversity and should therefore complement and diversify the gene pool of advanced cultivars (Frankel and Soule 1981). Rice landraces exhibit extensive variation, but only some of this variation has been introduced into a few elite lines (Ram et al. 2007). Ebana et al. (2008) evaluated genetic and phenotypic diversity in Japanese japonica rice landraces and developed a small core collection. These Japanese rice landraces showed a wide range of variation in culm length, with lengths ranging from 68 to $143 \mathrm{~cm}$ (Ebana et al. 2008). As a possible explanation for this variation, Asano et al. (2007) reported that multiple alleles of $s d l$ generated part of the phenotypic variation in Japanese rice varieties. However, allelic differences in $s d l$ cannot explain all of the culm-length variation in Japanese rice varieties. Recently, Monden et al. (2009) developed mPing-SCAR (sequence-characterized amplified region) markers based on transposable element insertion sites derived from Gimbozu EG4, which contains a high frequency of mPing. They detected two culm-length quantitative trait loci (QTLs) that were different from $s d 1$ in recombinant inbred lines (RILs) of Gimbozu EG4 and 
Nipponbare. These QTLs might be partially responsible for culm-length variation in Japanese rice varieties.

The frequency of DNA polymorphisms among Japanese rice cultivars is extremely low due to the narrow range of nucleotide sequence polymorphisms. This formerly prevented genetic analyses, including QTL analysis, using the progeny from Japanese rice cultivars. Recently, a number of simple sequence repeats (SSRs) have been identified after the completion of the rice genome sequencing project (IRGSP 2005). This has allowed effective large-scale screening for polymorphic SSRs between any pair of Japanese cultivars and permitted QTL analyses of their progeny. Using SSRs that are polymorphic between Japanese cultivars, several QTL analyses have been completed for eating quality (Kobayashi and Tomita 2008, Wada et al. 2008, Takeuchi et al. 2008), grain quality (Tabata et al. 2007, Kobayashi et al. 2007), and flowering time (Matsubara et al. 2008) in a mapping population derived from crosses between Japanese cultivars.

We recently released a set of reciprocal backcrossed inbred lines (BILs) derived from a cross between the Japanese cultivars Nipponbare and Koshihikari (Matsubara et al. 2008). Nipponbare and Koshihikari originated from crosses among Japanese japonica rice landraces and their progenies during the 1950s in Japan. Koshihikari has weak lodging resistance due to its slender and slightly long culm, whereas Nipponbare has a shorter culm than Koshihikari and exhibits stronger lodging resistance. Sasaki et al. (2002) and Ashikari et al. (2005) reported that Nipponbare and Koshihikari both commonly possess a functional (normal) $s d 1$ allele. This suggests that loci other than $s d 1$ might be involved in the differences in culm length between Nipponbare and Koshihikari. However, it is not yet clear whether the culm-length QTLs in Nipponbare and Koshihikari populations are the same QTLs reported by Monden et al. (2009).

The objective of the present study was to detect novel QTLs for culm length in Japanese rice varieties. We found QTLs for culm length and lower-internode lengths using a set of reciprocal BILs derived from crosses between Nipponbare and Koshihikari. We defined the genomic region of the QTLs, including that of one novel QTL, $q C L 1$, on chromosome 1 that was stably detected in multiple seasons and reciprocal populations, by means of substitution mapping using a series of substitution lines (SLs). $q C L 1$ appears to be different from several previously reported dwarf and semidwarf genes. We also investigated the distribution of the SSR alleles near the QTL region and variations in culm length in many Japanese rice landraces. Based on these results, we discuss the importance of the novel QTL for introducing short culm length and improving plant architecture during practical rice breeding in Japan.

\section{Materials and Methods}

\section{Plant materials}

Two populations of BILs were used for the QTL analysis: 79 BILs derived from the Nipponbare/Koshihikari//
Nipponbare cross (N-BILs) and 127 from the Nipponbare/ Koshihikari//Koshihikari cross (K-BILs) (Matsubara et al. 2008).

We used four semi-dwarf mutants and $s d l$ isogenic lines of Koshihikari, Hokuriku 100 (Samoto and Kanai 1975), Saga 1 (Nonaka et al. 1991), Koshihikari Tsukuba SD1 (Wang et al. 2005), and Hikarishinseiki (Tomita 2009), to compare culm and internode lengths with those of Koshihikari.

To evaluate the distribution of the QTL alleles in Japanese japonica rice landraces, we used the small core collection developed by Ebana et al. (2008), but we excluded two landraces (Akage and Fukoku) due to their aberrant shortculm phenotypes, which result from extremely early heading. We also used 11 additional Japanese landraces obtained from the National Institute of Agrobiological Science (NIAS) Genebank: Asahi (JP7416), Kamenoo (JP6521), Mii (JP7582), Ouba (JP7084), Sekitori (JP7911), Sen-ichi (JP6028), Shiratama (JP7846), Takenari (JP8043), Jukkoku (JP10577), Asahi (JP6761), and Awaakamai (JP7998). We used a total of 61 Japanese rice landraces in this study.

\section{QTL analysis in the N-BILs and K-BILs}

We cultivated the N-BILs in 2005 and the K-BILs in 2006 in an experimental field at the National Institute of Crop Science (NICS; Yawara, Tsukuba, Ibaraki, Japan). We also cultivated the N-BILs in 2006 and 2007 and the K-BILs in 2007 in an experimental field at NIAS (Kannondai, Tsukuba, Ibaraki, Japan). At the NICS field, seeds were sown in early May, and seedlings were transplanted into the paddy field at the end of May in plots with one row per line with a distance of $15 \mathrm{~cm}$ between plants and $30 \mathrm{~cm}$ between rows. At the NIAS field, seeds were sown in mid-April, and seedlings were transplanted into the paddy field in mid-May in plots with double row per line with a distance of $18 \mathrm{~cm}$ between plants and $30 \mathrm{~cm}$ between rows. We measured the mature lengths of the main culm (from the soil surface to the neck of the panicle) in five plants for each line.

We performed linkage mapping using version 3.0 of MAPMAKER/EXP (Lander et al. 1987), and used the Kosambi function to calculate genetic distances. We performed QTL analyses using composite interval mapping, as implemented by the Zmapqtl program (model 6) provided by version 2.5 of the QTL Cartographer software (Basten et al. $2005)$. We used genome-wide threshold values $(\alpha=0.05)$ to detect putative QTLs based on the results of 1000 permutations.

\section{Development of substitution lines (SLs) and characteriza- tion of the QTL on chromosome 1}

We developed three SLs to define the genomic region of the QTL on chromosome 1, $q C L 1$, and characterize their effects. An $F_{1}$ plant of Nipponbare/Koshihikari and its progenies were consecutively backcrossed three or four times with Koshihikari. In every backcrossed generation, plants heterozygous at the target region were selected using SSR markers (as described below). In the $\mathrm{BC}_{3} \mathrm{~F}_{3}$ and $\mathrm{BC}_{4} \mathrm{~F}_{3}$ 
generations, we performed whole-genome genotyping to select plants in which the target regions were homozygous for the Nipponbare allele and other regions were homozygous for the Koshihikari allele. For marker-assisted selection to develop the three SLs, we selected 102 SSR markers that covered the 12 chromosomes from the lists provided by McCouch et al. (2002), IRGSP (2005) and Matsubara et al. (2008). We added seven SSR markers (RM6887, RM10081, RM7278, RM1320, RM10132, RM3740, and RM10183) that showed polymorphisms between Nipponbare and Koshihikari on the short arm of chromosome 1 from IRGSP (2005). The DNA extraction and polymorphism detection procedures for the SSR markers are described by Matsubara et al. (2008).

The Koshihikari SLs were cultivated in an experimental field at NIAS in 2007 and 2008. The difference in culm length between Nipponbare and Koshihikari was small, and culm length and internode length were both affected by differences in environmental conditions. Thus, we planted each SL in one row and Koshihikari as the control line in another row in plots with double-row per line. At maturity, the main culms of 12 individuals from each line were collected. All internodes lengths longer than $0.5 \mathrm{~cm}$ and panicle length were measured, and mean values were calculated for each line. We then compared the mean internode and panicle lengths with those of the neighboring Koshihikari control line using the unequal-variance $t$-test procedure provided by the JMP 6.0 software (SAS Inc., Cary, NC).

\section{Sequence analysis of the dwarf genes $\mathrm{d} 18$ and $\mathrm{d} 2$}

To verify sequence differences between two dwarf genes ( $d 18$ and $d 2$ ) that are located in the same genomic region with the target QTL on chromosome 1, we amplified genomic fragments of Nipponbare and Koshihikari by means of polymerase chain reaction (PCR) using primers designed from the genomic sequences of the two genes (Itoh et al. 2001, Hong et al. 2003). We directly sequenced the amplified fragments $(0.5$ to $1.0 \mathrm{kbp})$ from both sides with appropriate primers using an ABI 3100 DNA sequencer (Applied Biosystems, Foster City, CA).

Distribution of SSR alleles near the QTL and phenotypic evaluation of Japanese rice landraces

We genotyped the Japanese rice landraces using SSR markers located on the short arm of chromosome 1 . The DNA extraction and PCR amplification procedures for the SSR markers are described by Matsubara et al. (2008). We fluorescently labeled the PCR products, and determined their fragment sizes using the ABI 3100 DNA sequencer and the Data Collection 2.0 and GeneMapper 3.7 software (Applied Biosystems). We cultivated the Japanese rice landraces in an experimental field at NIAS in 2007. At maturity, we measured all internode lengths of the main culms of five individuals per line. We then compared the mean culm length and internode lengths between the Nipponbare allele group and the group with other alleles at each DNA marker around the QTL using the unequal-variance $t$-test procedure of the JMP 6.0 software.

\section{Results}

Detection of QTLs for culm length in the N-BILs and K-BILs

Figure 1 shows the phenotypic variation in culm length of the N-BILs and K-BILs. The culm lengths (mean \pm SD) of Nipponbare were $79.1 \pm 2.7 \mathrm{~cm}$ at NICS in $2005,86.1 \pm 3.9$ $\mathrm{cm}$ at NIAS and $93.7 \pm 3.4 \mathrm{~cm}$ at NICS in 2006 , and $74.5 \pm 2.5$ $\mathrm{cm}$ at NIAS in 2007. The culm lengths of Koshihikari were $83.6 \pm 2.9 \mathrm{~cm}$ at NICS in $2005,91.2 \pm 3.4 \mathrm{~cm}$ at NIAS and $97.1 \pm 2.5 \mathrm{~cm}$ at NICS in 2006 , and $78.8 \pm 1.7 \mathrm{~cm}$ at NIAS in 2007. Nipponbare thus showed a shorter culm length than Koshihikari in both fields and in all three seasons. Culm lengths varied among years and between fields in both $\mathrm{N}$ BILs and K-BILs. Culm lengths of the N-BILs ranged from 68 to $94 \mathrm{~cm}$ in 2005, from 73 to $100 \mathrm{~cm}$ in 2006, and from 61 to $90 \mathrm{~cm}$ in 2007. Culm lengths of the K-BILs ranged from 75 to $106 \mathrm{~cm}$ in 2006 and from 59 to $88 \mathrm{~cm}$ in 2007 . Both populations showed a continuous distribution for culm length and contained transgressive segregants with values larger and smaller than those of the crossing parents, Nipponbare and Koshihikari. More number of transgressive lines had a culm length shorter than that of Nipponbare. The culm lengths (mean $\pm \mathrm{SD}$ ) of BILs for all seasons were 77.3 $\pm 4.6 \mathrm{~cm}, 82.0 \pm 4.5 \mathrm{~cm}$, and $73.5 \pm 5.2 \mathrm{~cm}$ in the N-BILs, and $90.1 \pm 5.4 \mathrm{~cm}$ and $74.9 \pm 5.0 \mathrm{~cm}$ in the K-BILs. All of the Pearson's correlation coefficients for the culm lengths between years were significant: 0.59 (2005-2006), 0.80 (2005-2007), and 0.64 (2006-2007) in the N-BILs, and 0.81 (2006-2007) in the K-BILs.

We detected two, one, and two QTLs for culm length $(q C L)$ in the N-BILs in 2005, 2006, and 2007, respectively (Fig. 2 and Table 1). The QTLs were mapped on chromosomes 1 ( $q C L 1$; near SSR marker RM8068) and 4 ( $q C L 4$; near RM7181) in 2005, on chromosome 1 ( $q C L 1$; near $\mathrm{RM} 8068$ ) in 2006, and on chromosomes 1 ( $q C L 1$; near $\mathrm{RM} 8068)$ and 3 (qCL3-2; near S87C10-17) in 2007. In the K-BILs, four and three QTLs for culm length were detected in 2006 and 2007, respectively. The QTLs were mapped on chromosomes 1 ( $q C L 1$; near RM8068), 3 ( $q C L 3-1$; near S0040-07), 3 ( $q C L 3-2$; near S87C10-17), and 7 ( $q C L 7$; near RM1364) in 2006, and on chromosomes 1 ( $q C L 1$; near RM8068), 3 ( $q C L 3-2$; near S87C10-17) and 7 ( $q C L 7$; near RM1364) in 2007. qCL1 was detected in the same chromosomal region in both BILs in all growing seasons and most likely represents the same locus. The additive effects and proportion of the variances explained by qCL1 (Table 1) were $-2.6 \mathrm{~cm}$ and $18.4 \%(2005),-3.0 \mathrm{~cm}$ and $27.9 \%$ (2006), and $-2.1 \mathrm{~cm}$ and $10.6 \%(2007)$ in the N-BILs, and they were $-2.3 \mathrm{~cm}$ and $12.3 \%$ (2006) and $-1.9 \mathrm{~cm}$ and $10.0 \%$ (2007) in the K-BILs. The Nipponbare allele of $q C L 1$ therefore decreased culm length.

Using RM8068, we divided both BILs into three subgroups: homozygous for the Nipponbare allele, homozygous 

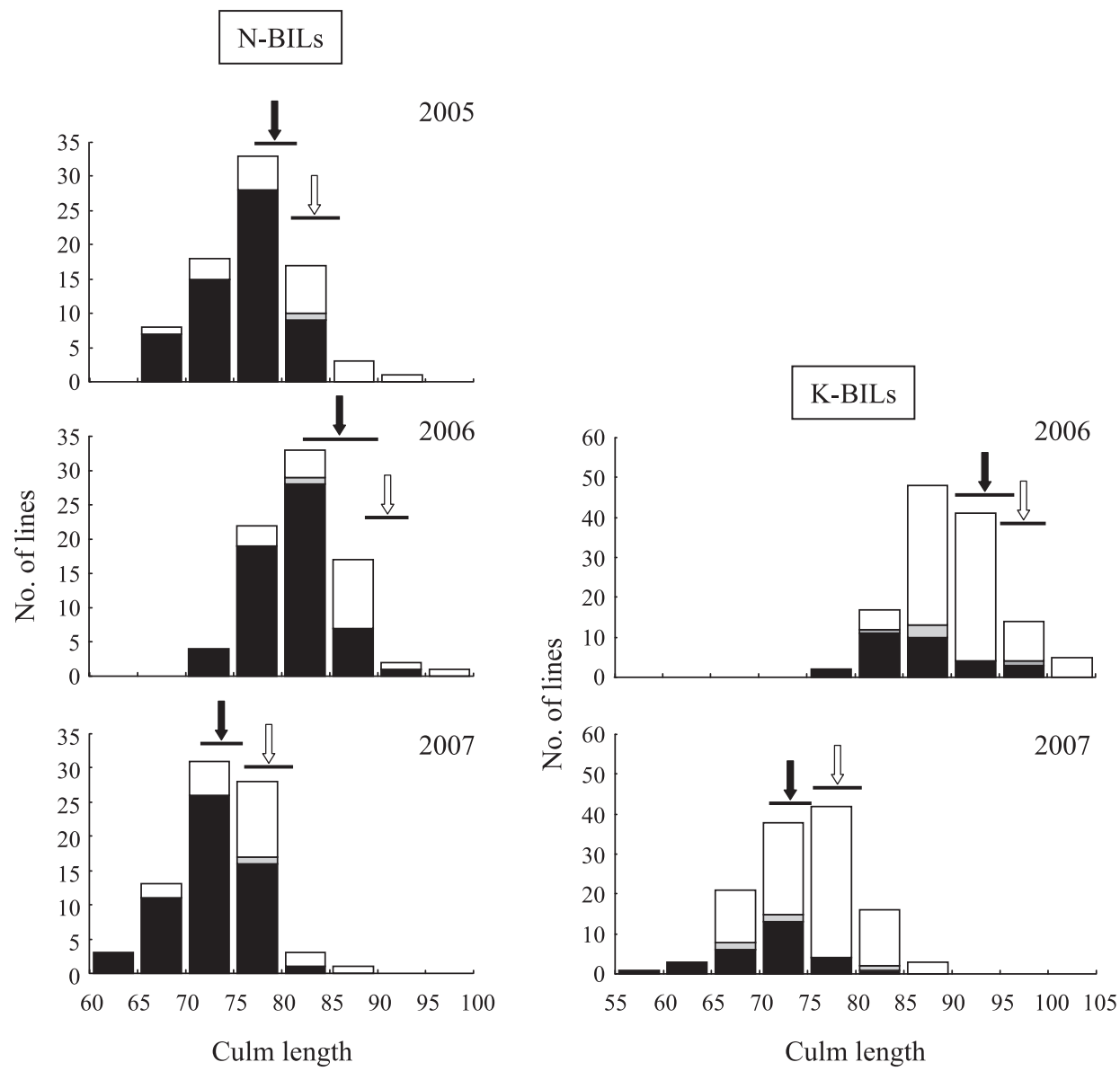

Fig. 1. Frequency distributions for culm length in 2005, 2006, and 2007 in the Nipponbare/Koshihikari//Nipponbare backcrossed inbred lines (NBILs), and in 2006 and 2007 in the Nipponbare/Koshihikari//Koshihikari BILs (K-BILs). Black and white arrows indicate the mean values for Nipponbare and Koshihikari, respectively. Horizontal lines under the arrows indicate the standard deviations. Black, white, and shaded bars indicate the number of lines homozygous for the Nipponbare allele, homozygous for the Koshihikari allele, and heterozygous, respectively, using SSR marker RM8068, which is the marker nearest to $q C L 1$, the culm-length QTL on the short arm of chromosome 1.

for the Koshihikari allele, and heterozygous (Fig. 1). The Nipponbare-homozygous group exhibited decreased culm length in both BILs in all seasons, although BILs that possessed the homozygous Nipponbare alleles showed a wide range of culm lengths. $q C L 3-2$ was detected in both BILs (in 2007 in the N-BILs, and in 2006 and 2007 in the K-BILs), and the Nipponbare allele increased culm length in both BILs. $q C L 7$ was only detected in the K-BILs but was found in both 2006 and 2007. qCL4 was detected in 2005 in the NBILs and $q C L 3-1$ was detected in 2006 in the K-BILs. The Nipponbare alleles of $q C L 7, q C L 4$, and $q C L 3-1$ all decreased culm length. Detection of these minor QTLs depended on differences in genetic backgrounds or seasons.

\section{Fine mapping of qCL1 using substitution lines}

$q C L 1$ was stably detected in both BILs in all seasons, but the estimated proportions of the variances explained and the additive effects were too small to explain the total phenotypic variation of the two BILs. To confirm the genetic effect of $q C L 1$ and its chromosome location, we adopted a more accurate evaluation method: each line was planted in one row and Koshihikari was planted as a control line in the adjacent row of double-row plots. We developed three Koshihikari SLs that possessed different Nipponbare segments in chromosome 1 by means of marker-assisted selection using 109 SSRs covering the rice genome. Koshihikari SL-1, SL-2, and SL-3 were substituted for Nipponbare homozygous at the marker intervals RM6887-RM3873 (0.2 to 6.2 Mbp in Rice Genome Sequence build 4.0; IRGSP 2005), RM6887RM10132 (0.2 to 2.6 Mbp), and RM10132-RM3873 (2.6 to $6.2 \mathrm{Mbp}$ ) on the short arm of chromosome 1 (Fig. 3). The culm lengths of Nipponbare, Koshihikari SL-1, and Koshihikari SL-2 were significantly shorter than those of the control lines (Koshihikari), whereas Koshihikari SL-3 did not differ significantly from the control line. Koshihikari SL-1 and SL-2 had culm lengths 6.0 and $4.1 \mathrm{~cm}$ shorter than the corresponding control line (Koshihikari), which supports the additive effects that were estimated using the BILs. The lowerinternode lengths were also shorter in Koshihikari SL-1 and SL-2 (data not shown). Thus, $q C L 1$ appears to be localized within $2.6 \mathrm{Mbp}$ on the rice genome sequence in a region of the short arm of chromosome 1 distal from RM10132. 
N-BILs

Chr 1

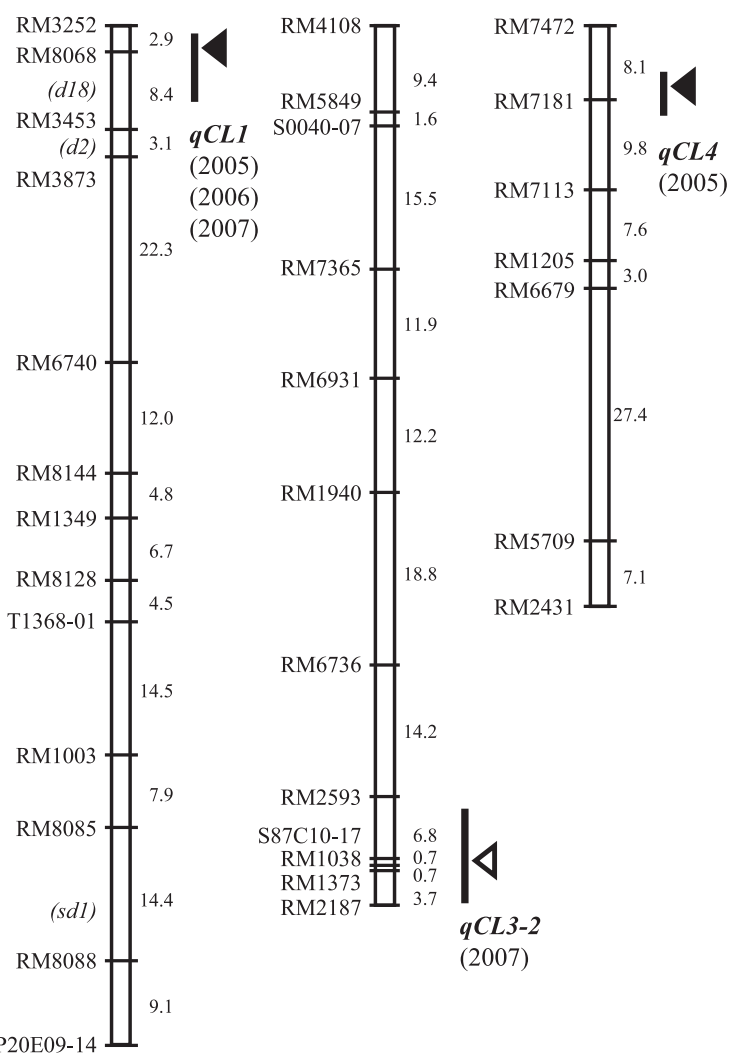

K-BILs
Chr 3

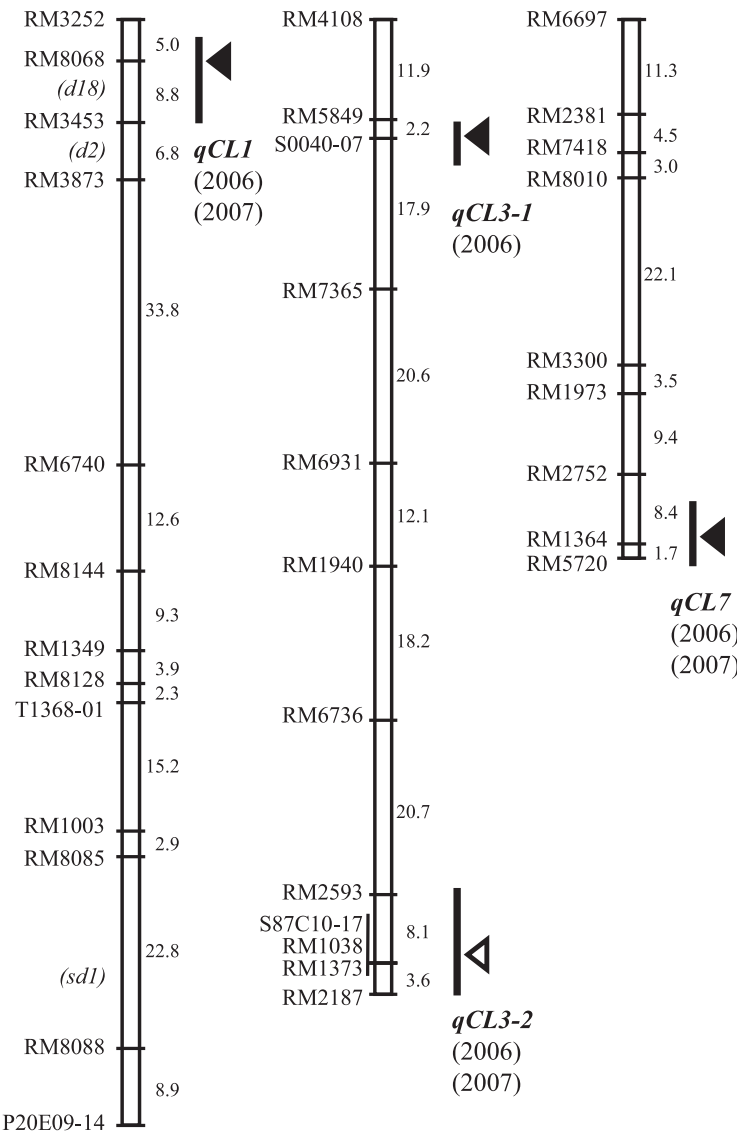

Fig. 2. Putative QTLs for culm length detected in Nipponbare/Koshihikari//Nipponbare backcrossed inbred lines (N-BILs) and Nipponbare/ Koshihikari//Koshihikari BILs (K-BILs). Parallel lines under the chromosome number denote the linkage map constructed for the two BILs (Matsubara et al. 2008). Marker names and genetic distance for each marker are indicated on the left and right sides of the linkage groups, respectively. Physical positions of dwarf and semi-dwarf genes $d 18, d 2$ and $s d 1$ were shown on the left side in parentheses on the linkage groups of chromosome 1. Vertical bars to the right of each linkage map indicate the $95 \%$ confidence intervals for the QTLs with a one-LOD reduction, and triangles indicate the positions of the LOD peaks at each QTL. Black and white triangles indicate that the Nipponbare alleles decrease and increase the trait score, respectively.

\section{Sequence analysis for $\mathrm{d} 18$ and $\mathrm{d} 2$}

Several dwarf or semi-dwarf genes were reported on chromosome 1 by Ogi et al. (1993) and Yoshimura et al. (1997). The genes $d 18$ and $d 2$ are located at 4.0 and $5.2 \mathrm{Mbp}$ from the distal end of the short arm of chromosome 1, respectively (Itoh et al. 2001, Hong et al. 2003). Although $q C L 1$ is apparently located at a different genome position from these genes, we determined genomic sequences of about $8.6 \mathrm{kbp}$ of $d 18$ and $8.1 \mathrm{kbp}$ of $d 2$ in Nipponbare and Koshihikari to exclude the possibility of additive effects by these genes. We found one synonymous substitution in the first exon and one SSR polymorphism in the upstream sequence of $d 18$ between Nipponbare and Koshihikari. Genomic sequences at all exons and introns of $d 2$ were the same in both Nipponbare and Koshihikari. We observed no nonsynonymous substitutions, insertions, or deletions in the genome sequences of $d 18$ and $d 2$ between Nipponbare and Koshihikari.

Culm lengths and internode lengths of the Koshihikari SLs, mutants, and isogenic lines

Figure 4 illustrates the distribution patterns of all internode lengths and panicle length for the main culm of Koshihikari, Nipponbare, Koshihikari SL-1, three sdl isogenic lines of Koshihikari (Koshihikari Tsukuba SD1, Hikarishinseiki, and Saga 1), and the semi-dwarf mutant cultivar Hokuriku 100 in 2007. Semi-dwarfism of Hokuriku 100 is controlled by the single recessive gene, $d 60$ (Tanisaka et al. 1990). Culm length of Nipponbare, Koshihikari SL-1, the $s d l$ isogenic lines of Koshihikari and the semi-dwarf 
Table 1. Quantitative trait loci (QTLs) for culm length detected in two groups of backcrossed inbred lines (BILs): Nipponbare/Koshihikari// Nipponbare (N-BILs) and Nipponbare/Koshihikari//Koshihikari (K-BILs)

\begin{tabular}{|c|c|c|c|c|c|c|c|c|}
\hline Population & Year & QTL & Marker interval & Nearest marker & Chromosome & LOD & PVE & $\mathrm{AE}$ \\
\hline \multirow[t]{5}{*}{ N-BILs } & \multirow[t]{2}{*}{2005} & $q C L 1$ & RM3252-RM3453 & RM8068 & 1 & 5.2 & 18.4 & -2.6 \\
\hline & & $q C L 4$ & RM7472-RM7113 & RM7181 & 4 & 3.0 & 10.6 & -1.7 \\
\hline & 2006 & $q C L 1$ & RM3252-RM3453 & RM8068 & 1 & 6.4 & 27.9 & -3.0 \\
\hline & \multirow[t]{2}{*}{2007} & $q C L 1$ & RM3252-RM3453 & RM8068 & 1 & 3.3 & 10.6 & -2.1 \\
\hline & & $q C L 3-2$ & RM2593-RM1038 & S87C10-17 & 3 & 3.4 & 13.2 & 2.0 \\
\hline \multirow[t]{7}{*}{ K-BILs } & \multirow[t]{4}{*}{2006} & $q C L 1$ & RM3252-RM3453 & RM8068 & 1 & 5.2 & 12.3 & -2.3 \\
\hline & & $q C L 3-1$ & RM5849-RM7365 & S0040-07 & 3 & 4.6 & 13.6 & -2.4 \\
\hline & & $q C L 3-2$ & RM2593-RM1038 & S87C $10-17$ & 3 & 9.3 & 24.1 & 3.4 \\
\hline & & $q C L 7$ & RM2752-RM5720 & RM1364 & 7 & 3.2 & 6.3 & -1.8 \\
\hline & \multirow[t]{3}{*}{2007} & $q C L 1$ & RM3252-RM3453 & RM8068 & 1 & 4.0 & 10.0 & -1.9 \\
\hline & & $q C L 3-2$ & RM2593-RM1038 & S87C $10-17$ & 3 & 5.3 & 13.6 & 2.4 \\
\hline & & $q C L 7$ & RM2752-RM5720 & RM1364 & 7 & 3.4 & 10.8 & -2.1 \\
\hline
\end{tabular}

All genetic parameters were calculated using version 2.5 of QTL Cartographer (Basten et al. 2005).

${ }^{a} \log$-likelihood value.

${ }^{b}$ percentage of total phenotypic variance explained by the QTL.

${ }^{c}$ additive effect of the Nipponbare allele.

LOD thresholds used for the detection of the QTL in composite interval mapping: 2.6 in 2005, 2.8 in 2006, and 2.7 in 2007 for the N-BILs; 3.0 in 2006 and 2.8 in 2007 for the K-BILs.

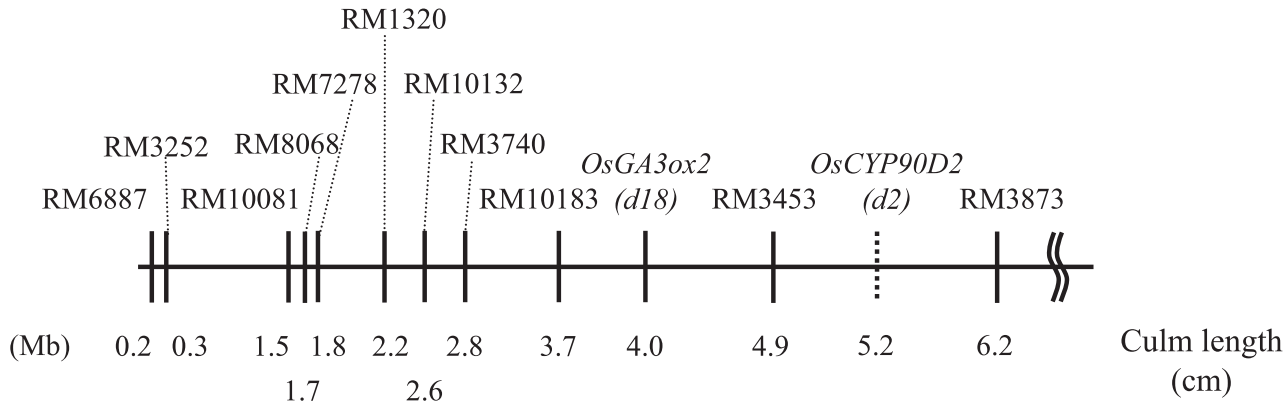

Nipponbare

Koshihikari

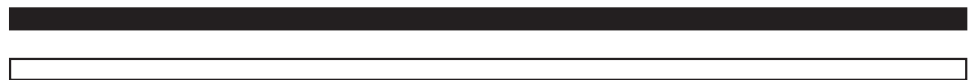

$76.3+2.9 * *$

$81.3+5.8$

Koshihikari SL-1

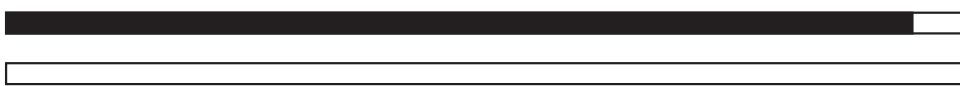

$80.3+4.9 * *$

Koshihikari

$86.3+5.0$

Koshihikari SL-2

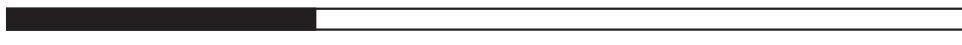

$85.7+2.6 *$

Koshihikari

Koshihikari SL-3

$89.6+3.6$

Koshihikari

$90.0+2.7$

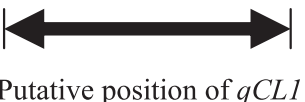

Fig. 3. Illustration of loci on chromosome 1 and of the culm lengths of Nipponbare, of Koshihikari (control), and of three Koshihikari substitution lines (SLs). Marker positions of the simple sequence repeat (SSR) markers, and dwarf genes OsGA3ox2 (d18) and OsCYP90D2 (d2) in the rice genome sequence (Rice Genome Sequence build 4.0; IRGSP 2005) are oriented from the short arm (left) to the long arm (right). Black and white bars indicate homozygous for the Nipponbare and Koshihikari alleles, respectively. ${ }^{*}$ and $* *$ indicate significantly different culm lengths $(P<0.05$ and $P<0.01$, respectively) between Nipponbare or the Koshihikari SLs and the corresponding control value (for Koshihikari). 


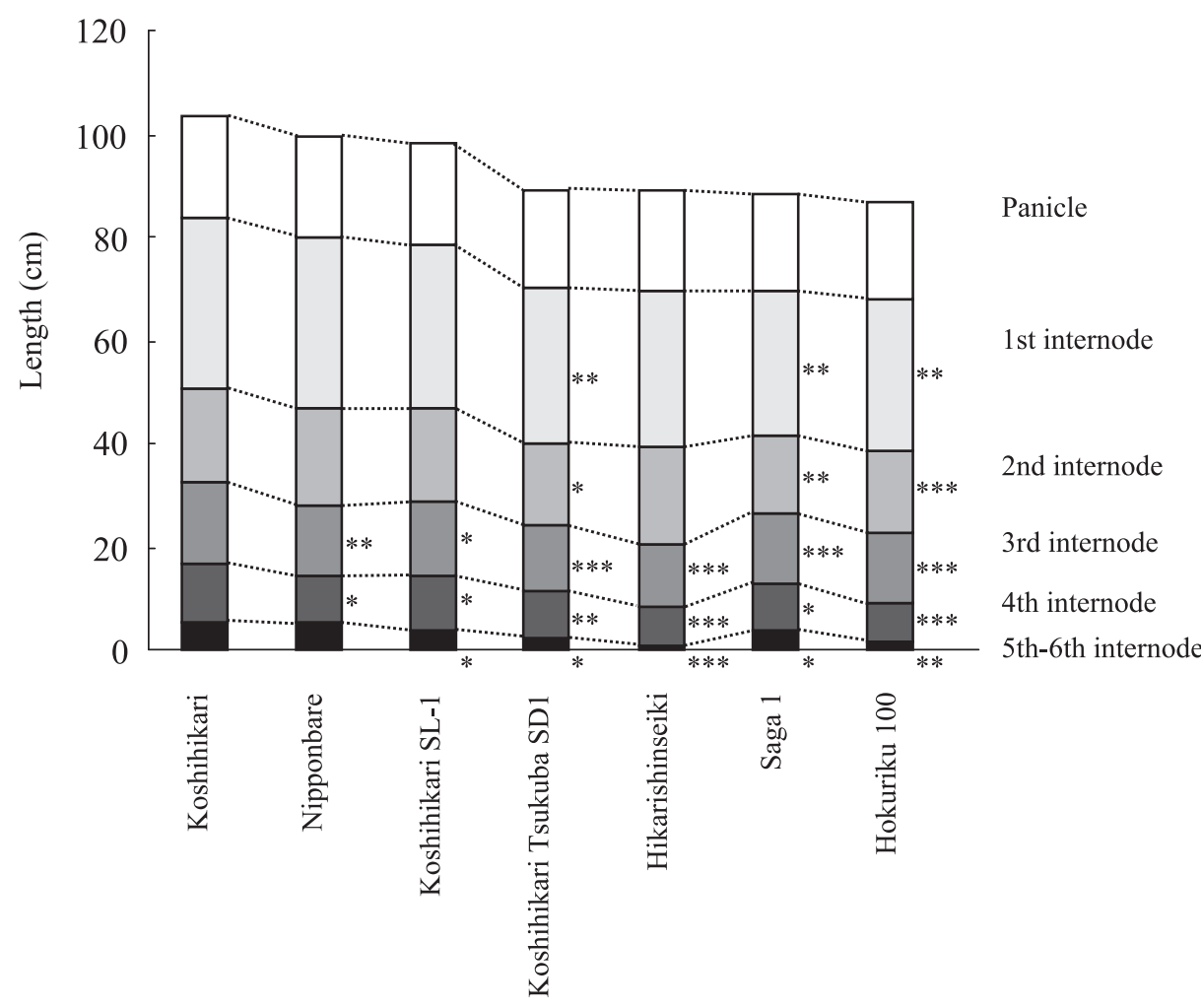

Fig. 4. Diagram of the panicle, 1st internode, 2nd internode, 3rd internode, 4th internode, and 5th-6th internode lengths of Koshihikari, Nipponbare, Koshihikari SL-1, and Koshihikari isogenic lines with a semi-dwarf phenotype. *, **, and *** indicate significantly different lengths from the corresponding length for the control (Koshihikari) at $P<0.05, P<0.01$, and $P<0.001$, respectively.

mutant cultivar were shorter than Koshihikari. The 3rd and 4th internode lengths of Nipponbare were $14.0 \pm 0.9 \mathrm{~cm}$ and $9.3 \pm 1.8 \mathrm{~cm}$ long, and they were significantly shorter than those of Koshihikari. The 3rd, 4th, and 5th-6th internode lengths of Koshihikari SL-1 with the Nipponbare allele of $q C L 1$ were $14.1 \pm 1.6 \mathrm{~cm}, 10.5 \pm 1.8 \mathrm{~cm}$, and $3.4 \pm 1.9 \mathrm{~cm}$, and they were significantly shorter than those of Koshihikari. Koshihikari Tsukuba SD1, Saga 1, and Hokuriku 100 all had significantly shorter internodes than those of Koshihikari. Hikarishinseiki had significantly shorter 3rd to 6th internodes than Koshihikari. The dwarfism effect of Koshihikari SL-1 with the Nipponbare allele of $q C L 1$ was weak compared to the mutant gene effects of the semi-dwarf mutant cultivar and the $s d 1$ isogenic lines. However, $q C L 1$ only shortened the lower internodes, and it didn't significantly decrease the 1 st and 2 nd internode lengths or the panicle length.

Correlation between genotypes around the QTL and culm lengths in Japanese rice landraces

To reveal how much of the total phenotypic variance for culm length can be explained by $q C L 1$ in the Japanese landraces, we investigated the distribution of the Nipponbare $q C L 1$ allele for short culm length in 61 Japanese landraces. Since landraces with the Nipponbare $q C L 1$ allele show Nipponbare alleles at DNA markers near $q C L 1$ on the short arm of chromosome 1, we surveyed the genotypes of the 61 landraces using 11 SSR markers and one nucleotide substitu- tion in $d 18$, which is located near $q C L 1$. All of the SSR markers exhibited multiple alleles; the fewest number of alleles was two at RM3252 and the largest number of alleles was eight at RM3873. When the landraces were divided into two groups based on the genotype (i.e., the Nipponbare allele group and the "other alleles" group), the mean culm lengths for the Nipponbare allele group and the other alleles group differed significantly at five SSR markers (Table 2): by $7.0 \mathrm{~cm}$ at RM6887, $10.9 \mathrm{~cm}$ at RM8068, $8.1 \mathrm{~cm}$ at $\mathrm{RM} 7278,8.8 \mathrm{~cm}$ at RM1320, and $11.0 \mathrm{~cm}$ at RM10132. The average culm lengths in the Nipponbare allele group were shorter than those in the other alleles group. When the average culm lengths of all landraces in the group with no Nipponbare allele were compared, there was no significant difference for any of the SSR markers (data not shown).

Differences in the lengths of the 3rd to 6th internodes between Nipponbare and the other allele group were also significant at the same five SSR markers (Table 2): by $5.5 \mathrm{~cm}$ at $\mathrm{RM} 6887,6.1 \mathrm{~cm}$ at RM8068, $4.3 \mathrm{~cm}$ at $\mathrm{RM} 7278,4.8 \mathrm{~cm}$ at RM1320, and $5.2 \mathrm{~cm}$ at RM10132. Figure 5 shows the variation in culm length among the 61 Japanese rice landraces plus Nipponbare and Koshihikari in 2007. The 11 landraces with the Nipponbare allele at RM8068 had culm lengths that ranged from 76.1 to $95.5 \mathrm{~cm}$, whereas those of the other landraces ranged from 75.1 to $119.8 \mathrm{~cm}$. Mean culm lengths in the Nipponbare allele group were clearly smaller than that in the other alleles group. 
Table 2. Comparison of average culm length and the length of the 3rd to 6th internodes between the Nipponbare allele group and the other allele group in 61 Japanese rice landraces

\begin{tabular}{|c|c|c|c|c|c|c|c|c|c|}
\hline \multirow[b]{2}{*}{ Marker name } & \multirow{2}{*}{$\begin{array}{l}\text { Position } \\
\text { (Mbp) }\end{array}$} & \multicolumn{2}{|c|}{ Number of landraces } & \multicolumn{3}{|c|}{ Culm length $(\mathrm{cm})$} & \multicolumn{3}{|c|}{ Length of 3rd to 6th internodes $(\mathrm{cm})$} \\
\hline & & $\begin{array}{l}\text { Nipponbare } \\
\text { allele }\end{array}$ & $\begin{array}{l}\text { Other } \\
\text { alleles }\end{array}$ & $\begin{array}{l}\text { Nipponbare } \\
\text { allele }\end{array}$ & Other alleles & $t$-value & $\begin{array}{l}\text { Nipponbare } \\
\text { allele }\end{array}$ & Other alleles & $t$-value \\
\hline RM6887 & 0.2 & 15 & 46 & $89.3 \pm 2.7$ & $96.3 \pm 1.5$ & $2.28 *$ & $28.4 \pm 3.6$ & $33.9 \pm 6.0$ & $3.31 * *$ \\
\hline RM3252 & 0.3 & 21 & 40 & $95.6 \pm 2.1$ & $92.6 \pm 2.2$ & 0.97 & $32.7 \pm 5.5$ & $31.1 \pm 4.1$ & 1.04 \\
\hline RM10081 & 1.5 & 31 & 30 & $93.1 \pm 1.9$ & $96.1 \pm 2.0$ & 1.10 & $32.4 \pm 7.0$ & $32.6 \pm 4.8$ & 0.09 \\
\hline RM8068 & 1.7 & 11 & 50 & $85.6 \pm 2.8$ & $96.5 \pm 1.4$ & $3.47 * * *$ & $27.6 \pm 4.1$ & $33.7 \pm 5.8$ & $3.48 * * *$ \\
\hline RM7278 & 1.8 & 12 & 49 & $88.6 \pm 3.1$ & $96.7 \pm 1.8$ & $2.28 *$ & $29.2 \pm 6.5$ & $33.5 \pm 5.9$ & $2.13 *$ \\
\hline RM1320 & 2.2 & 9 & 52 & $87.1 \pm 3.4$ & $95.9 \pm 1.4$ & $2.36^{*}$ & $28.4 \pm 5.2$ & $33.2 \pm 5.9$ & $2.34 *$ \\
\hline RM10132 & 2.6 & 9 & 52 & $85.4 \pm 3.9$ & $96.4 \pm 1.5$ & $2.62 *$ & $28.2 \pm 4.5$ & $33.4 \pm 6.1$ & $2.18 *$ \\
\hline RM3740 & 2.8 & 14 & 47 & $92.1 \pm 3.4$ & $95.0 \pm 1.7$ & 0.75 & $29.2 \pm 4.4$ & $33.2 \pm 6.3$ & 1.92 \\
\hline RM10183 & 3.7 & 33 & 28 & $93.9 \pm 1.9$ & $96.7 \pm 2.3$ & 0.92 & $32.3 \pm 5.3$ & $33.2 \pm 7.1$ & 0.53 \\
\hline OsGA3ox2(d18) & 4.0 & 57 & 4 & $95.9 \pm 1.5$ & $85.4 \pm 5.3$ & 1.91 & $33.2 \pm 6.2$ & $28.1 \pm 1.6$ & 1.63 \\
\hline RM3453 & 4.8 & 22 & 39 & $92.2 \pm 2.3$ & $96.9 \pm 2.0$ & 1.51 & $30.7 \pm 5.0$ & $34.2 \pm 6.7$ & 2.01 \\
\hline RM3873 & 6.2 & 15 & 46 & $91.8 \pm 2.2$ & $94.2 \pm 1.8$ & 0.66 & $31.6 \pm 5.5$ & $32.6 \pm 6.2$ & 0.54 \\
\hline
\end{tabular}

$*^{* * *}$, and *** indicate markers that differ significantly between the Nipponbare allele group and the other alleles group at $P<0.05, P<0.01$, and $P<0.001$, respectively $(t$-test).

\section{Discussion}

Identifying useful new semi-dwarf genes is important for the genetic manipulation of plant architecture in practical rice breeding. Previously, it has been difficult to perform genetic analysis among Japanese cultivars due to the low frequency of DNA polymorphism. Using information on more than 18,000 SSR sequences identified by the rice genome sequencing project (IRGSP 2005), we detected five QTLs in the two reciprocal BILs between the Japanese rice cultivars Nipponbare and Koshihikari (Table 1 and Fig. 2). A QTL for culm length on chromosome $1(q C L 1)$ was detected in all three years (Fig. 2), indicating that $q C L 1$ is a stable QTL. The Nipponbare allele at this QTL shortened from 1.9 to $3.0 \mathrm{~cm}$ of culm length, and proportion of phenotypic variance explained were from 10.0 to $27.9 \%$ in the QTL analysis. Because culm length varied among years and between fields, we adopted a more accurate two-row evaluation method for culm and internode lengths. As a result, we could finely map $q C L 1$ within about $2.6 \mathrm{Mbp}$ at marker interval RM6887RM10132 using the Koshihikari substitution lines (SLs) and explain some of the difference in culm length between Nipponbare and Koshihikari (Fig. 4). It is important to note that the Nipponbare allele of $q C L 1$ shortened only the lower internode lengths.

$q C L 3-2$, which is located on the long arm of chromosome 3 , increased culm length as a Nipponbare allele (Table 1 and Fig. 2). Matsubara et al. (2008) reported a QTL with a large effect for heading date $(\mathrm{HdlO})$ in the same marker interval between RM2593 and RM1038. The Nipponbare allele of $H d 16$ increased the number of days to heading. This suggests that $q C L 3-2$ is affected pleiotropically by Hd16. $q C L 7$ was only detected in the K-BILs but was detected in both years. The expression of $q C L 7$ might depend on differences in the rice genetic backgrounds. $q C L 3-1$ and $q C L 4$ were found in one year or one population. The difference in culm length between Nipponbare and Koshihikari might thus result from a combination of five QTLs: $q C L 1, q C L 3-1, q C L 3-$ 2, $q C L 4$, and $q C L 7$.

Kobayashi et al. (2003) reported a minor QTL for culm length or plant height near $q C L 1$. They used recombinant inbred lines (RILs) derived from an indica cultivar (Milyang 23) and a japonica cultivar (Akihikari), and found that the Akihikari allele shortened culm length. Since Akihikari shares some of its ancestral varieties with Nipponbare, it is possible that Akihikari possesses the same alleles as Nipponbare at $q C L 1$. However, the Akihikari QTL allele has not yet been proven to be identical to either $d 18$ or $d 2$. Thus, we hypothesize that the $q C L 1$ detected in the present study is a novel QTL. Monden et al. (2009) used RILs derived from a cross between the Japanese japonica lines Gimbozu EG4 and Nipponbare. They reported a culm-length QTL on the short arm of chromosome 3 and found that the Nipponbare allele decreased culm length. This QTL might thus be the same as $q C L 3-1$, because the Nipponbare allele of $q C L 3-1$ also decreased culm length. Xiao et al. (1996) found one plant height QTL on the long arm of chromosome 7 in RILs of indica 9024/japonica LH422. This QTL might be the same as $q C L 7$ in the present study.

To reveal the relationship between $q C L 1$ and the dwarf and semi-dwarf genes on chromosome 1, we determined the genomic sequences of $d 18$ and $d 2$ in Nipponbare and Koshihikari. Itoh et al. (2001) mapped $d 18$ to the short arm of chromosome 1, where it encodes gibberellin $3 \beta$-hydroxylase (OsGA3ox2). Hong et al. (2003) mapped the brassinosteroiddeficient dwarf mutant $d 2$ to the short arm of chromosome 1 , where the gene, CYP90D2, encodes a cytochrome P450. There were no nonsynonymous polymorphisms of the genomic sequences of $d 18$ and $d 2$ between Nipponbare and Koshihikari in the present study. Substitution mapping using 
Koshihikari SLs showed that the position of $q C L 1$ had different marker intervals from those of $d 18$ and $d 2$ (Fig. 3), suggesting that $q C L 1$ is a novel culm-length QTL but that the $d 18$ and $d 2$ alleles are not. $s d 1$ is located on the long arm of chromosome 1 and encodes gibberellin 20-oxydase, OsGA20ox2 (Sasaki et al. 2002). Sasaki et al. (2002) and Ashikari et al. (2005) indicated that the $s d l$ alleles of Nipponbare and Koshihikari were the functional (normal) type. No QTLs were detected near $s d 1$ in both the BILs in the present study. Based on these results, we conclude that $s d l$ was not responsible for the culm length differences between Nipponbare and Koshihikari.

Some gibberellin-related mutants, including those of $d 18$ and $s d 1$, show dwarfism, with short lower internodes (Sakamoto et al. 2004), whereas brassinosteroid-related mutants, including $d 2$, show inhibition of elongation at the second internode, erect leaves, and photomorphogenesis in the dark (Hong et al. 2003). Koshihikari SLs with the Nipponbare genomic segment around $q C L 1$ exhibited shorter lower internodes than those of Koshihikari (Fig. 4). The phenotypic appearance and distribution pattern of $q C L 1$ are thus more similar to those of the $d 18$ and $s d 1 \mathrm{mu}-$ tants associated with the gibberellin pathway than to those of $d 2$ mutants associated with the brassinosteroid pathway. There are no metabolic enzyme genes and signaling pathway genes for gibberellins and brassinosteroids on the short arm of chromosome 1 (Sakamoto et al. 2004, 2006). Fine mapping of $q C L 1$ is currently in progress. Cloning and molecular identification of $q C L 1$ would provide an opportunity to understand a novel factor involved in the phytohormone biosynthesis and signaling pathways for the phytohormones.

The mean culm length of the Japanese landraces differed significantly between the Nipponbare allele group and the other alleles group at SSR markers RM8068, RM7278, RM1320, and RM10132 (Table 2). Japanese rice landraces with the Nipponbare allele at the four SSR markers were collected from various geographical regions of Japan (Ebana et al. 2008). The results suggest that the Nipponbare allele at $q C L 1$ was originally distributed in the Japanese rice landraces and has subsequently been used to develop Nipponbare and other short-culm cultivars in Japan. Four landraces (Asahi (JP7416), Asahi (6761), Kyoutoasahi, and Aichiasahi) have the Nipponbare alleles at all the four SSRs (data not shown), indicating that these landraces might be ancestors of the short-culm allele of Nipponbare. Investigation of SSR alleles of recently released rice cultivars will help us to understand the importance of $q C L 1$ in practical rice breeding in Japan.

The semi-dwarf $q C L 1$ allele of Nipponbare could explain part of the culm-length variation in Japanese landraces (Fig. 5). However, the landraces without the Nipponbare allele at RM8068 showed a wide range of culm lengths. QTLs other than $q C L 1$ should thus be responsible for some of the variation in culm length in Japanese rice landraces. Asano et al. (2007) reported multiple dwarf alleles of $s d 1$ in Japanese rice cultivars. Monden et al. (2009) reported two culm-

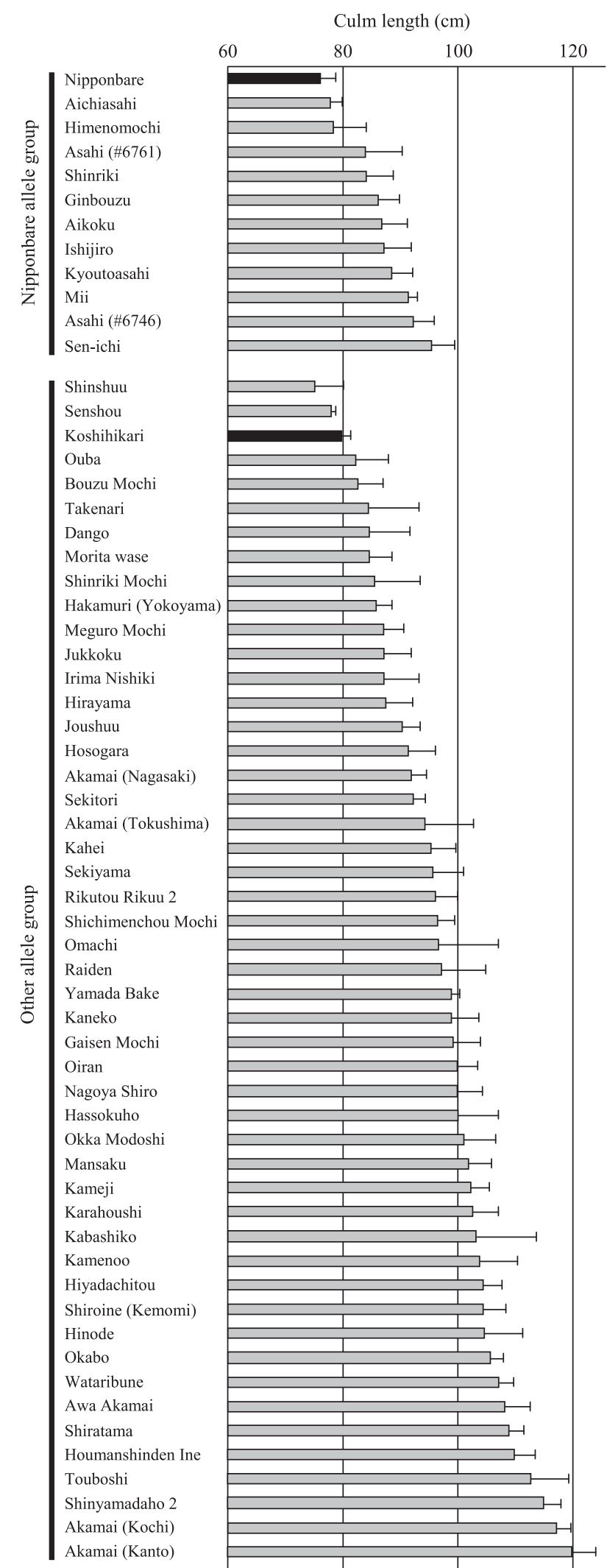

Fig. 5. Variation in the culm lengths of 61 Japanese rice landraces in 2007. The Japanese rice landraces were divided into two groups: those with the Nipponbare allele and those with other alleles at the SSR marker RM8068. Horizontal range bars indicate standard deviations. Black bars indicate the culm lengths of Nipponbare and Koshihikari. 
length QTLs on chromosomes 3 and 10 in RILs of Japanese rice lines. Differences in the heading dates of these landraces also influence the culm-length variation. In the present study, one SSR marker (RM6887) at the distal region of the short arm of chromosome 1 differed significantly between the Nipponbare allele group and the other alleles group (Table 2). These loci would therefore contribute to the variation in culm length among the landraces that lacked the Nipponbare allele at RM8068. Moreover, several dwarf mutants and cultivars have been generated from natural mutations (Sasaki et al. 2002, Itoh et al. 2004). Association mapping or linkage disequilibrium mapping would be a promising tool to identify the QTLs and genes responsible for natural variation in the germplasm accessions because it does not require crossed populations or pedigree information. To reveal how many QTLs are associated with the variation in culm length in Japanese rice landraces, genome-wide association mapping (Flint-Garcia et al. 2003, Aranzana et al. 2005, Iwata et al. 2007) using the Japanese rice landraces will be necessary.

The identification of agronomically important QTLs and pyramiding of these QTLs represents a useful strategy for efficient crop breeding. Several QTLs with relatively large phenotypic effects have been cloned by following a mapbased strategy (reviewed by Yamamoto et al. 2009). The combination of desirable alleles of the genes isolated by this analysis could improve defective plant architectures and may have increased grain filling (Ashikari et al. 2005, Sakamoto et al. 2006, Takeda and Matsuoka 2008). Although the phenotypic effect of $q C L 1$ was small compared with that of $s d 1$ alleles, the short lower internodes produced by the Nipponbare allele of $q C L 1$ should contribute to strong lodging resistance in rice. The combination of $q C L 1$ with other morphogenetic genes, including genes for semidwarfism, number of grains, grain width, and tiller angle, will provide useful information for fine-tuning of plant architecture in most Japanese rice varieties.

\section{Acknowledgments}

The authors are grateful to Mr. Yuzo Minobe (Plant Genome Center Co., Ltd.) for providing seeds of Koshihikari Tsukuba SD1, Dr. Motonori Tomita (Tottori University) for providing seeds of Hikarishinseiki, and Mr. Yuji Hirota (Saga Prefecture Agriculture Research Center) for providing seeds of Saga 1. We also thank Dr. Toshiyuki Takai and Mr. Yasunori Nonoue for their excellent technical assistance during the measurement of internode lengths and in developing the SLs.

\section{Literature Cited}

Aranzana, M.J., S.Kim, K.Zhao, E. Bakker, M.Horton, K. Jakob, C.Lister, J.Molitor, C.Shindo, C.Tang et al. (2005) Genome-wide association mapping in Arabidopsis identifies previously known flowering time and pathogen resistance genes. PLoS Genet. 1:e60. Asano,K., T.Takashi, K, Miura, Q.Qian, H.Kitano, M.Matsuoka and
M.Ashikari (2007) Genetic and molecular analysis of utility of $s d 1$ alleles in rice breeding. Breed. Sci. 57: 53-58.

Ashikari, M., H. Sakakibara, S. Lin, T. Yamamoto, T. Takashi, A.Nishimura, E.R.Angeles, Q.Qian, H.Kitano and M.Matsuoka (2005) Cytokinin oxidase regulates rice grain production. Science 309: 741-745.

Basten,C.J., B.S.Weir and Z.B.Zeng (2005) QTL cartographer, version 1.17. Department of Statistics, North Carolina State University, Raleigh, p. 189.

Ebana,K., Y.Kojima, S.Fukuoka, T.Nagamine and M.Kawase (2008) Development of mini core collection of Japanese rice landrace. Breed. Sci. 58: 281-291.

Flint-Garcia,S.A., J.M.Thornsberry, S.Edward and I.Buckler (2003) Structure of linkage disequilibrium in plants. Annu. Rev. Plant Biol. 54: 357-374.

Frankel,O.H. and M.E.Soule (1981) Conservation and Evolution. Cambridge Univ. Press, New York, p. 366.

Hedden,P. (2003) The genes of the Green Revolution. Trends Genet. 19: 5-9.

Hong,Z., M.Ueguchi-Tanaka, K.Umemura, S.Uozu, S.Fujioka, S. Takatsuto, S.Yoshida, M.Ashikari, H.Kitano and M.Matsuoka (2003) A rice brassinosteroid-deficient mutant, ebisu dwarf (d2), is caused by a loss of function of a new member of cytochrome P450. Plant Cell 15: 2900-2910.

IRGSP (2005) The map-based sequence of the rice genome. Nature 436: 793-800.

Itoh,H., M.Ueguchi-Tanaka, N.Sentoku, H.Kitano, M.Matsuoka and M.Kobayashi (2001) Cloning and functional analysis of two gibberellin 3 beta-hydroxylase genes that are differently expressed during the growth of rice. Proc. Natl. Acad. Sci. USA 98: 89098914.

Itoh,H., T.Tatsumi, T.Sakamoto, K.Otomo, T.Toyomasu, H.Kitano, M.Ashikari, S.Ichihara and M.Matsuoka (2004) A rice semi-dwarf gene, Tan-Ginbozu (D35), encodes the gibberellin biosynthesis enzyme, ent-kaurene oxidase. Plant Mol. Biol. 54: 533-547.

Iwata,H., Y.Uga, Y.Yoshioka, K.Ebana and T.Hayashi (2007) Bayesian association mapping of multiple quantitative trait loci and its application to the analysis of genetic variation among Oryza sativa L. germplasms. Theor. Appl. Genet. 114: 1437-1449.

Kinoshita,T. (1998) Linkage mapping using mutant genes in rice. Rice Genet. Newsl. 15: 13-74.

Kinoshita, T. and N.Shinbashi (1982) Identification of dwarf genes and their character expression in the isogenic background. Jpn. J. Breed. 32: 219-231.

Kobayashi,A., B.Genliang, Y.Shenghai and K.Tomita (2007) Detection of quantitative trait loci for white-back and basal-white kernels under high temperature stress in japonica rice varieties. Breed. Sci. 57: 107-116.

Kobayashi,A. and K. Tomita (2008) QTL detection for stickiness of cooked rice using recombinant inbred lines derived from crosses between japonica rice cultivars. Breed. Sci. 58: 419-426.

Kobayashi,S., Y.Fukuta, T.Sato, M.Osaki and G.S.Khush (2003) Molecular marker dissection of rice (Oryza sativa L.) plant architecture under temperate and tropical climates. Theor. Appl. Genet. 107: $1350-1356$

Lander, E.S., P.Green, J.Abrahamson, A. Barlow, M.J. Daly, S.E. Lincoln and L.Newburg (1987) Mapmaker: an interactive computer package for constructing primary genetic linkage maps of experimental and natural populations. Genomics 1: 174-181.

Matsubara, K., I. Kono, K.Hori, Y.Nonoue, N. Ono, A. Shomura, T.Mizubayashi, S. Yamamoto, U.Yamanouchi, K. Shirasawa et al. 
(2008) Novel QTLs for photoperiodic flowering revealed by using reciprocal backcross inbred lines from crosses between japonica rice cultivars. Theor. Appl. Genet. 117: 935-945.

McCouch,S.R., L.Teytelman, Y.Xu, K.B.Lobos, K.Clare, M.Walton, B.Fu, R.Maghirang, Z.Li, Y.Xing et al. (2002) Development and mapping of 2240 new SSR markers for rice (Oryza sativa L.). DNA Res. 9: 257-279.

Monden, Y., K.Naito, Y.Okumoto, H.Saito, N.Oki, T.Tsukiyama, O.Ideta, T.Nakazaki, S.R.Wessler and T.Tanisaka (2009) High potential of a transposon mPing as a marker system in japonica $\times$ japonica cross in rice. DNA Res. 16: 131-140.

Monna,L., N.Kitazawa, R.Yoshino, J.Suzuki, H.Masuda, Y.Maehara, M.Tanji, M.Sato, S.Nasu and Y.Minobe (2002) Positional cloning of rice semidwarfing gene, $s d-1$ : rice "green revolution gene" encodes a mutant enzyme involved in gibberellin synthesis. DNA Res. 9: 11-17.

Nonaka,K., Y.Takagi, S.Matsuyuki, H.Yokoh, Y.Hirota, T.Shigyo, O.Shigetomi, H.Kishikawa, D. Nakamura, H.Kanayama et al. (1991) A new rice cultivar "Saga 1 Gou". Bull. Saga Pref. Agric. Exp. Stn. 27: 1-19.

Ogi,Y., H.Kato, K.Maruyama, A.Saito and F.Kikuchi (1993) Identification of RFLP markers closely linked to the semidwarfing gene at the $s d-1$ locus in rice. Jpn. J. Breed. 43: 141-146.

Ram,S.G., V.Thiruvengadam and K.K.Vinod (2007) Genetic diversity among cultivars, landraces and wild relatives of rice as revealed by microsatellite markers. J. Appl. Genet. 48: 337-345.

Sakamoto, T., K. Miura, H. Itoh, T. Tatsumi, M. Ueguchi-Tanaka, K.Ishiyama, M.Kobayashi, G.K.Agrawal, S.Takeda, K.Abe et al. (2004) An overview of gibberellin metabolism enzyme genes and their related mutants in rice. Plant Physiol. 134: 1642-1653.

Sakamoto,T., Y.Morinaka, T.Ohnishi, H. Sunohara, S. Fujioka, M. Ueguchi-Tanaka, M.Mizutani, K.Sakata, S.Takatsuto, S. Yoshida et al. (2006) Erect leaves caused by brassinosteroid deficiency increase biomass production and grain yield in rice. Nature Biotech. 24: 105-109.

Samoto, S. and D.Kanai (1975) Studies on the mutation breeding in rice. I. short stiff mutations induced by gamma-ray irradiation to the rice variety Koshihikari. Jpn. J. Breed. 25: 1-7.

Sasaki, A., M. Ashikari, M.Ueguchi-Tanaka, H.Itoh, A.Nishimura, D.Swapan, K.Ishiyama, T.Saito, M.Kobayashi, G.S.Khush et al. (2002) Green revolution: a mutant gibberellin-synthesis gene in rice. Nature 416: 701-702.

Spielmeyer, W., M.H.Ellis and P.M.Chandler (2002) Semidwarf $(s d-1)$, "green revolution" rice, contains a defective gibberellin 20- oxidase gene. Proc. Natl. Acad. Sci. USA 99: 9043-9048.

Tabata,M., H.Hirabayashi, Y.Takeuchi, I.Ando, Y.Iida and R.Ohsawa (2007) Mapping of quantitative trait loci for the occurrence of white-back kernels associated with high temperatures during the ripening period of rice (Oryza sativa L.). Breed. Sci. 57: 47-52.

Takahashi,M. and K. Takeda (1969) Type and grouping of internode pattern in rice culm - Genetical studies on rice plant, XXXVII- . Mem. Fac. Agric. Hokkaido Univ. 7: 32-43.

Takeda,K. and M.Takahashi (1969) Preliminary report on cross experiments in six types of internode pattern in rice culm-Genetical studies on rice plant, XXXVIII-. Mem. Fac. Agric. Hokkaido Univ. 7: 44-50.

Takeda,S. and M.Matsuoka (2008) Genetic approaches to crop improvement: responding to environmental and population changes. Nature Rev. Genet. 9: 444-457.

Takeuchi,Y., K. Hori, K. Suzuki, Y.Nonoue, Y.Takemoto-Kuno, H.Maeda, H.Sato, H.Hirabayashi, H.Ohta, T.Ishii et al. (2008) Major QTLs for eating quality of an elite Japanese rice cultivar, Koshihikari, on the short arm of chromosome 3. Breed. Sci. 58: 437-445.

Tanisaka,T., M.Tomita and H.Yamagata (1990) Gene analysis for the semidwarfism of two mutant strains, Hokuriku 100 and Kanto 79, induced from a rice variety Koshihikari: studies on the utility of artificial mutations in plant breeding XVIII. Jpn. J. Breed. 40: 103117.

Tomita, M. (2009) Introgression of Green Revolution $s d l$ gene into isogenic genome of rice super cultivar Koshihikari to create novel semidwarf cultivar 'Hikarishinseiki' (Koshihikari-sd1). Field Crops Res. (in press).

Wada,T., T.Ogata, M.Tsubone, Y.Uchimura and Y.Matsue (2008) Mapping of QTLs for eating quality and physicochemical properties of the japonica rice 'Koshihikari'. Breed. Sci. 58: 427-435.

Wang,Z.X., S.Sakaguchi, Y.Oka, N.Kitazawa and Y.Minobe (2005) Breeding of semi-dwarf Koshihikari by using genome breeding method. Breed. Res. 7 (Suppl. 1, 2): 217.

Xiao,J., J.Li, L.Yuan and S.D.Tanksley (1996) Identification of QTLs affecting traits of agronomic importance in a recombinant inbred population derived from a subspecific rice cross. Theor. Appl. Genet. 92: 230-244.

Yamamoto,T., J.Yonemaru and M.Yano (2009) Towards the understanding of complex traits in rice: substantially or superficially? DNA Res. 16: 141-154.

Yoshimura,A., O.Ideta and N.Iwata (1997) Linkage map of phenotype and RFLP markers in rice. Plant Mol. Biol. 35: 49-60. 